\title{
Conservative Therapy with Intrapleural Urokinase Avoids Primary Thoracotomy in Childhood Necrotizing Pneumonia: A Case Series
}

\author{
Karniza Khalid ${ }^{1}$, Kean Hong Eng ${ }^{2}$, Nurul Amani Abd Ghani ${ }^{2}$, Mohan Arunasalam Nallusamy ${ }^{3}$ \\ ${ }^{1}$ Clinical Research Centre and ${ }^{2}$ Department of Paediatrics, Hospital Tuanku Fauziah, Kangar, Perlis, Ministry of Health Malaysia; \\ ${ }^{3}$ Department of Surgery, Hospital Sultanah Bahiyah, Alor Setar, Kedah, Ministry of Health Malaysia.
}

\section{Corresponding Author:}

Dr. Karniza Khalid

Email: karniza.khalid@moh.gov.my

This is an Open Access article distributed under the terms of the Creative Commons Attribution License (creativecommons.org/ licenses/by/3.0).

Received : April 20, 2019

Accepted : August 1, 2019

Published : August 10, 2019

\begin{abstract}
Introduction: Necrotizing pneumonia is a rare complication of community-acquired pneumonia. Lung resection is gaining attention as therapeutic alternative in patients who fail to respond to antibiotics. However, we aimed to describe two cases of childhood necrotizing pneumonia successfully managed conservatively. Case Report: MA, a 2-year old boy presented with acute onset respiratory distress with 5 days history of fever and cough. Computed tomography $(\mathrm{CT})$ thorax revealed right pleural effusion with features of necrotizing pneumonia. Despite chest drainage and two weeks of broad-spectrum antibiotic therapy, there was no clinical improvement. Intrapleural urokinase instillation resulted in clinical and radiological improvement. Our second case was NR, an 8-month old girl who presented with acute onset breathing difficulty. CT thorax reported severe left lower lobe necrotizing pneumonia with left hydro-pneumothorax. Ultrasound of left thorax showed presence of left pleural effusion despite nil chest drainage. She improved clinically and radiologically following intrapleural urokinase administration over three days. Conclusion: Intrapleural urokinase may prevent the need of thoracotomy or videoassisted thoracoscopic surgery in necrotizing pneumonia.
\end{abstract}

Keywords: Drainage, Pneumonia, Respiratory System, Thoracotomy, Urokinase-Type Plasminogen Activator.

\section{Introduction}

Necrotizing pneumonia is a rare and severe complication of bacterial community-acquired pneumonia [1,2] where pleural involvement is almost universal [3]. It only represents $0.8 \%$ of community-acquired pneumonia but may be seen upto $6 \%$ of hospitalized communityacquired pneumonia [4]. Necrotizing pneumonia is characterized by the destruction of the lung parenchyma resulting in multiple thin-walled cavities. Additionally, it is often accompanied by the formation of empyema and bronchopleural fistulae [4]. Necrotizing pneumonia is associated with high morbidity and mortality [5]. However, the lack of clinical and local guidelines resulted in indistinct management for these patients. The findings of necrotizing pneumonia on the first radiological assessment almost always warrants for a surgical intervention. Lung resection is commonly acknowledged as the therapeutic alternative in patients who fail to respond to antibiotics and continues to deteriorate despite antibiotics optimization, which is commonly used as the first-line of management.

We describe two pediatric cases of necrotizing pneumonia with the successful use of intrapleural urokinase instillation with subsequent clinical and radiological improvement, escaping the need for pleural debridement. 


\section{Case Reports}

\section{Case 1}

MA, a 2-year-old boy, who was previously healthy, presented to our centre with five days history of flu-like symptoms and shortness of breath. His inflammatory markers were markedly raised and the initial chest X-Ray (CXR) showed features of right lobar pneumonia [Fig.1a]. A course of intravenous ceftriaxone and azithromycin for 10 days resulted in little clinical improvement. CT thorax was subsequently done revealing right large pleural effusion with collapse consolidation of right upper lobe, suggesting the possibility of necrotizing pneumonia. Thus right intercostal drain was inserted. Unfortunately, despite two weeks on antibiotic therapy and escalation of treatment, there was no obvious clinical or radiological improvement. Follow-up ultrasound thorax showed right fibropurulent pleural effusion. Following paediatric surgical opinion, a decision for right intrapleural urokinase of 10,000 units twice daily instillation was given for three days. Drainage volume improved from $60 \mathrm{ml}$ to $100 \mathrm{ml}$ for the first two days post-urokinase instillation, draining mostly pus. His pleural fluid culture and sensitivity revealed no growth. Repeated CXR a week after urokinase instillation showed marked improvement with clearing up of right upper zone consolidation with resolved pleural effusion [Fig.1b,c]. In view of the positive outcome demonstrated both clinically and radiologically MA avoided the need for surgical intervention.
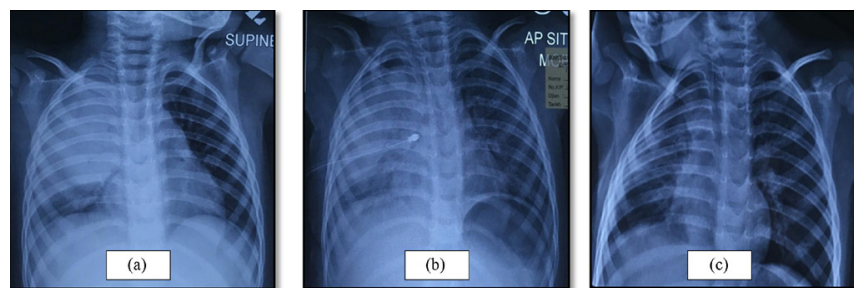

Fig.1: Chest $x$-rays showing progressive changes during in-patient care. (a): Chest $x$-ray on admission. (b): Day 7 on chest drainage prior to urokinase instillation. (c): Chest $x$-ray one week after urokinase instillation.
He completed intravenous cefepime for 14 days and intravenous cloxacillin therapy for four weeks before being discharged home. He was seen at our out-patient department a week later for review and was well and cheerful.

\section{Case 2}

NR was a previously well, 8-month old girl who was acutely ill on presentation with hypoxemia. She required non-invasive biphasic positive airway pressure (BiPAP) ventilation shortly after admission. Her first CXR [Fig.2a] revealed left lung consolidation and she was started on high dose intravenous cefuroxime. She responded poorly despite escalation of therapy. A repeated CXR revealed worsening of left lower lobe consolidation with pleural effusion and multiple cavitation. Left intercostal drain was inserted which drained thick pus. Pleural fluid culture and sensitivity revealed Staphylococcus aureus. Drainage was nil by day two of drain insertion of drain. However a repeat ultrasound of left hemithorax showed persistence of left pleural effusion. After
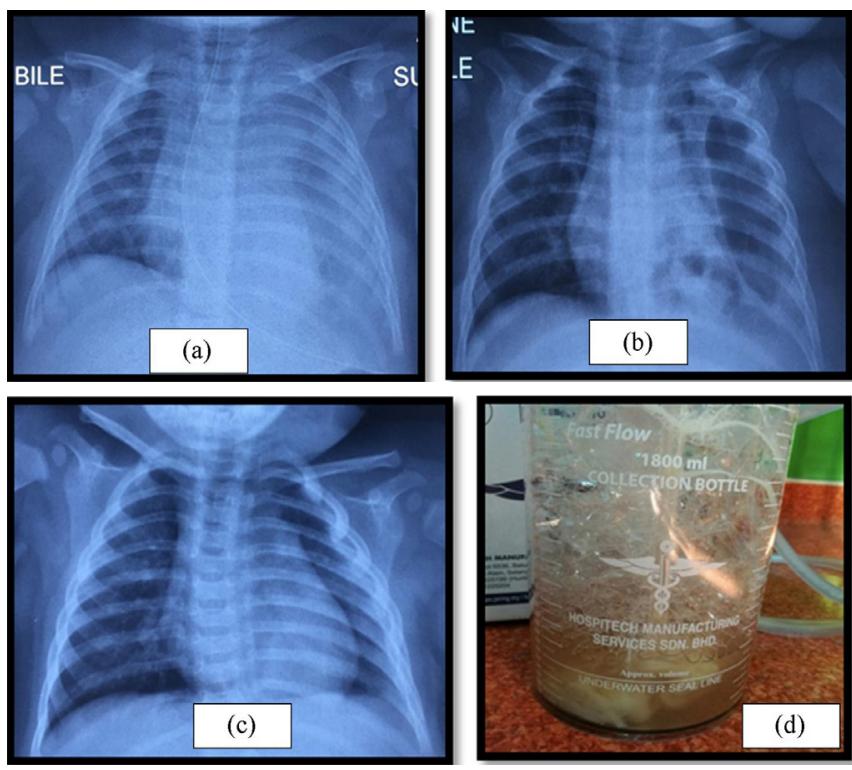

Fig.2: Chest $x$-rays showing progressive changes during inpatient care. (a): Chest $x$-ray on admission. (b): Loculated left pneumothorax with multiple pneumatocele. (c): Chest $x$-ray before discharge. (d): Fibropurulent pleural fluid collection after urokinase instillation. 
discussion with paediatric surgeon, intrapleural urokinase 10,000 units twice daily was given for three days. Unfortunately, her condition began to deteriorate three days later with chest recession and intermittent tachypnea. Repeated CXR at that point showed loculated left pneumothorax with multiple pneumatocoele formation [Fig.2b]. Contrastenhance computed tomography (CECT) of the thorax revealed severe left lower lobe necrotizing pneumonia with left hydropneumothorax. Left intercostal drain was re-inserted, draining thick pus [Fig.2d]. Her clinical condition and biochemical markers began to improve thereafter. After almost three weeks on BiPAP, she was successfully weaned down to nasal prong oxygen. Serial repeated CXRs showed clearing up of left lung consolidation and resolved pleural effusion [Fig.2c]. She completed intravenous cefepime therapy for 14 days, intravenous azithromycin for five days and intravenous cloxacillin for six weeks. NR was discharged well after 37 days of hospitalization. Intramuscular pneumococcal vaccine was given prior to discharge.

\section{Discussion}

Necrotizing pneumonia is a rare and serious complication of bacterial pneumonia. It involves loss of normal pulmonary architecture and parenchyma with the presence of thin-walled cavitating lesions [6]. A retrospective study conducted by Lemaitre et al. (2013) had witnessed a rise in the number of complicated pneumonia attributable to the H1N1 pandemic [6]. Due to its rarity in clinical contact, further researches to define its epidemiology and comparative studies among affected patients are deemed necessary to identify potential predictive factors to the development of necrotizing pneumonia, particularly in children.

Both of our cases demonstrated the clinical efficacy of medical treatment for necrotizing pneumonia through the instillation of intrapleural urokinase. The instilled urokinase acts by breaking down the extracellular DNA in the pleural space, thus reducing the fluid viscosity and enhancing drainage [7]. Furthermore, a study has found that pulmonary drainage together with the use of urokinase instillation is as effective as videoassisted thoracoscopic surgery as the first-line management of septated pulmonary disease in children [8]. Another similar report [3] also reported the safety and efficacious use of urokinase in treating two paediatric cases with pneumonia who failed to respond to optimized antibiotic treatment. Hence, there exists the prevailing perception that childhood necrotizing pneumonia is of a favourable outcome, even with conservative approach.

In the light of diagnostic modalities to confirm childhood necrotizing pneumonia, lung ultrasonography is assumed to correlate favourably well with chest computed tomography, especially in cases where the lung necrosis is massive [9]. Apart from its simple technique and reliability, tolerance of patient is one of the crucial factors to consider, especially when dealing with pediatrics population. However, if pulmonary gangrene is suspected, computed tomography of the thorax with contrast enhancement is deemed superior to evaluate the vascular supply [5] before any decision on surgical intervention is made.

Despite the growing evidence of promising conservative management in childhood necrotizing pneumonia, caution needs to be practiced when deciding for the optimal clinical care as most published reports were retrospective in nature, and there may be the presence of other interplay factors that may affect the efficacy and the result of the treatment, such as the varied immunization schedules according to different regions and the local antibiotic prescribing practices.

\section{Conclusion}

Our case demonstrated the safety and efficacy of intrapleural urokinase to facilitate drainage of a complicated parapneumonic effusion which is reasonably useful, particularly in children. It 
is as an effective measure despite the suspicion of necrotizing disease on initial imaging which conventionally warrants the need for surgical intervention. Thus, the timely use of intrapleural urokinase may potentially escape the need for primary thoracotomy.

Acknowledgement: The authors would like to thank the Director General of Health Malaysia for his permission to publish the paper.

Contributors: KK: primarily in literature review and manuscript writing; KHE, NAAG: manuscript editing, clinical patient management and clinical data retrieval; MAN: critical inputs into the finalized manuscript, final review prior to submission. KK will act as a study guarantor. All authors approved the final version of this manuscript.

Funding: None; Competing interests: None stated.

\section{References}

1. Tsai YF, Ku YH. Necrotizing pneumonia: a rare complication of pneumonia requiring special consideration. Curr Opin Pulm Med. 2012;18(3):246-252.

2. Spencer DA, Thomas MF. Necrotising pneumonia in children. Paediatric Respiratory Reviews. 2014;15(3):240-245.
3. Demirel N, Quizon A, Belteton DeLeon EL, Reiter $\mathrm{J}$, Colin AA. On the nature of pleural involvement in necrotizing pneumonia: A report of two cases of life threatening late complications. Pediatr Pulmonol. 2014; 49:e90-e95.

4. Masters IB, Isles AF, Grimwood K. Necrotizing pneumonia: an emerging problem in children? Pneumonia. 2017;9(1):11.

5. Chatha N, Fortin D, Bosma KJ. Management of necrotizing pneumonia and pulmonary gangrene: a case series and review of the literature. Canadian Respiratory Journal. 2014;21(4):239-245.

6. Lemaitre C, Angoulvant F, Gabor F, Makhoul J, Bonacorsi S, Naudin J. Necrotizing pneumonia in children: report of 41 cases between 2006 and 2011 in a French tertiary care center. Pediatr Infect Dis. 2013;32(10):1146-1149.

7. Pabary R, Balfour-Lynn IM. Complicated pneumonia in children. Breathe. 2013;9:210-222.

8. Marhuenda C, Barcelo C, Fuentes I, Guillen G, Cano I, Lopez M, et al. Urokinase versus VATS for treatment of empyema: A randomized multicenter clinical trial. J. Pediatr. 2014;134(5):e1301-e1307.

9. Lai SH, Wong KS, Liao SL. Value of lung ultrasonography in the diagnosis and outcome prediction of pediatric community-acquired pneumonia with necrotizing change. PLoS One. 2015;10(6):e0130082. 